\title{
Effects of supine body position and low radial accelerations on the visually perceived apparent zenith
}

\author{
CHRISTIAN RAPHEL, CORINNE CIAN, and PIERRE-ALAIN BARRAUD \\ Centre de Recherches du Service de Santé des Armées, La Tronche, France \\ and \\ CHRISTOPHE MICHEYL \\ Laboratoire Perception et Mécanismes Auditifs, CNRS, Lyon, France
}

\begin{abstract}
The visually perceived eye level (VPEL) has been shown to shift toward the lower part of the body in upright subjects facing toward the axis of rotation on a centrifuge. This shift occurs in the same direction as the shift in the gravito-inertial forces (Gis) produced by very low radial acceleration (centrifugation) combined with gravity. The purpose of this study was to determine whether the same phenomenon affects the visually perceived apparent zenith (VPAZ) in subjects in a supine position. Twelve supine subjects were instructed to set a luminous target to the VPAZ, either while they were in total darkness and motionless or while undergoing very low centrifugation. Data showed that Gis induced a VPAZ shift similar to that observed for the VPEL. Thus, as is the case for the VPEL, the corresponding logarithmic psychophysical function of the VPAZ may be considered to be a type of oculogravic illusion phenomenon with differences in the subjects' that differs from subject to subject, depending on the subject's sensitivity to low radial accelerations. Data on VPEL and VPAZ support the notion that the subjective perception of eye level in total darkness takes into account changes - even if extremely slight - in the direction of the gravito-inertial forces produced by the combination of gravity and low radial accelerations, although subjects are unaware of the $G i$ shift. However, depending on the intensity of the radial acceleration and the angular deviation of $G i$ relative to $G$, the shift of the VPEL and the VPAZ can be either amplified or attenuated. Moreover, differences between VPEL and VPAZ responses suggest two explanatory assumptions-namely, that this is (1) a peripheral phenomenon dependent on the neurophysiological anisotropy of the otolithic system or (2) a central phenomenon dependent on the relevance assigned to the peripheral information by the integrative sensory functions and the associative processes.
\end{abstract}

When an observer makes a spatial judgment, he/she can easily determine whether a given target is to the left or to the right or above or below the direction of his/her gaze. This ability to localize objects in space involves a coordinate framework organized in two principal subjective dimensions: the straight ahead for right versus left judgments, and the eye level for above versus below judgments (Matin \& Li, 1995).

For the usual direction of gravity and axis of the head, eye level can be referenced in several ways (Howard \& Templeton, 1966). The gravity referenced eye level (Stoper $\&$ Cohen, 1991), more generally called visually perceived eye level (VPEL), divides visually perceived space along a horizontal plane through the eyes that is perceived to be perpendicular to the direction of gravity. The head relative eye level (Stoper \& Cohen, 1991), also called the trans-

This research was supported by the Ministry of Defense: Délégation Générale pour l'Armement (DGA) and Service de Santé des Armées (SSA). Correspondence concerning this article should be addressed to C. Raphel, Unité de Psychologie, B.P. 87, 38702, La Tronche Cedex, France (e-mail: raphelchristian@ compuserve.com). verse plane of the eyes (TPE) by Howard and Templeton (1966), is the plane through the socket of the eye that is subjectively perceived as perpendicular to the head axis.

When the observer is erect - that is, standing or seated with head and body in the vertical position-these two VPEL and TPE planes correspond. When the subject rotates his/her head backward for a few degrees while keeping his/her pupil at the same level, his/her VPEL retains the same subjective reference-that is, the horizontal plane through the eyes-whereas the TPE is always perpendicular relative to the head axis (Figure 1A). When the subject rotates his/her whole body backward more$60^{\circ}$, for instance-he/she can no longer define the VPEL, which lies outside his/her visual field, whereas the TPE can still be subjectively perceived. When the tilt of the whole body is near the horizontal position, the subject can define both the TPE and the visually perceived apparent zenith (VPAZ), which is the plane through the eyes perceived as being parallel to the direction of gravity. When the subject is in a supine position-that is, the body and head axis is perpendicular to the direction of gravitythe VPAZ and the TPE correspond (Figure 1B). 


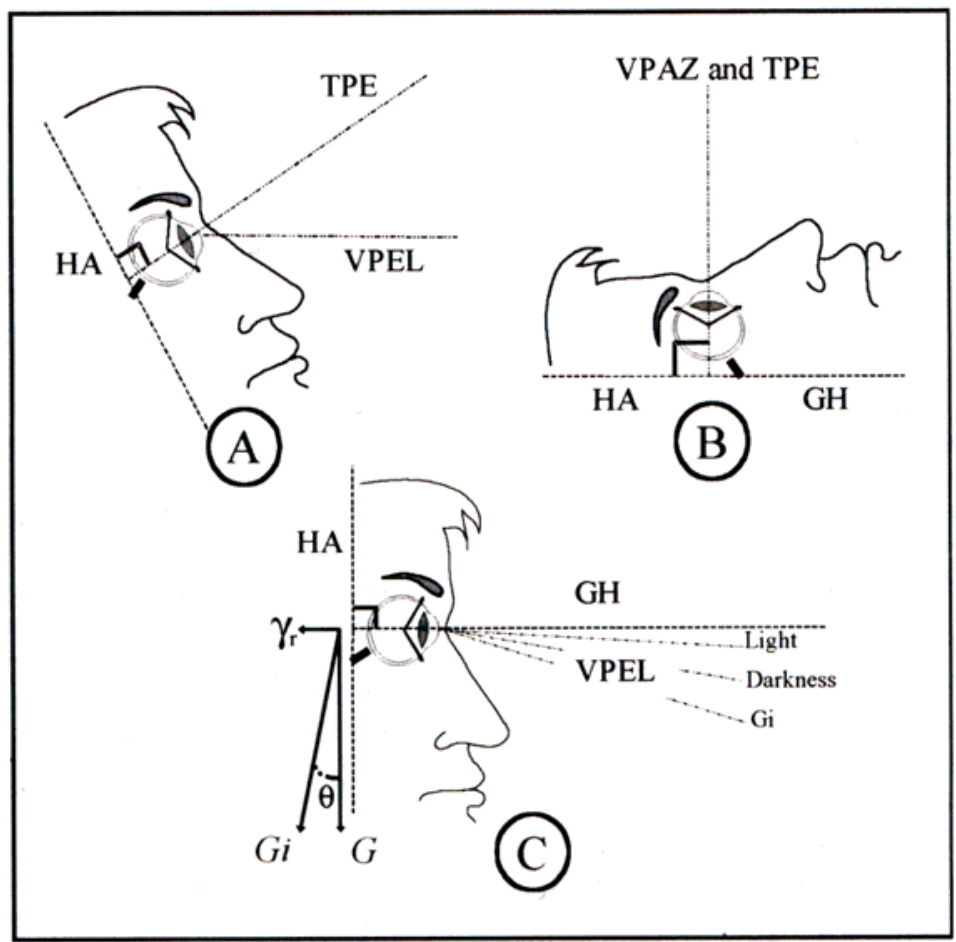

Figure 1. Different visually perceived subjective references according to gravity and head positions. Panel A shows the difference between the visually perceived eye level (VPEL), which is defined relative to the gravitational horizontal (GH), and the transverse plane of the eye (TPE), which is defined relative to the head axis (HA). In Panel B, when the head axis is horizontal in a supine position, the TPE and the visually perceived apparent zenith (VPAZ) correspond. Panel $C$ shows the variations of the VPEL according to perceptual conditions: (1) in an illuminated visual environment (Light), (2) in darkness and motionless (Darkness), and (3) in darkness with gravito-inertial force $G i$ produced by centrifugation.

Many studies concerning the visually perceived eye references have been reported for VPEL-that is, in upright subjects. In darkness, the VPEL is more difficult to set and less accurate-lying $3^{\circ}-6^{\circ}$ under the physical eye level - than in an illuminated environment, where the error is less than $3^{\circ}$ (Hoppeler, 1913; Matin, 1982, 1986; McDougall, 1903; Nair, 1958; Raphel \& Barraud, 1994; Raphel, Barraud, Koessler, \& Cian, 1996; Sharp, 1934; Stoper \& Cohen, 1986; Figure 1C). For subjects in a supine position, Cohen and Larson (1974) have shown that in darkness, the perceived morphological horizon is "below" Reid's baseline reference value-that is, between $4^{\circ}$ and $5^{\circ}$ toward the lower part of the body. This reference value corresponds to the perceived horizon in the upright position and total darkness (idem VPEL), which is approximately $6^{\circ}$ below the true morphological horizon (idem horizontal plane through the eyes), as is confirmed in the literature.

Our research focused on the eye level in darkness, where only extraretinal, vestibular, and other proprioceptive information is available to aid a subject in judging the perceived location of a visual target. More precisely, in dark surroundings, three kinds of information must be avail- able to the observer setting a luminous target to the VPEL: (1) extraretinal information regarding the orientation of the head relative to gravity, (2) extraretinal information regarding the orientation of the eye relative to the head, and (3) information regarding the location of a visual target on the retina (Matin \& Li, 1992, 1995; Stoper \& Cohen, 1986, 1989, 1991). This information and the associated sensory receptors are called the target/gravity system by Stoper and Cohen (1986).

Most of the studies concerning the orientation of the head relative to gravity show that the VPEL is displaced with the pitch of the head and in the same direction but that this displacement is smaller than the head displacement; sometimes, it occurs only for a head pitch of $20^{\circ}$ and more (Cohen, 1973, 1981; Correia, Hixon, \& Niven, 1968; Li \& Matin, 1993; Matin \& Li, 1995). According to some researchers, a drop in otolithic sensitivity-supposedly proportional to the head tilt-accounts for the weakness and variability of the phenomena (Howard, 1982, 1986; Schöne, 1964, 1975; Wade \& Schöne, 1971).

With regard to extraretinal information concerning the orientation of the eye relative to the head, it has been demonstrated that errors in localizing a luminous target 
at the place perceived as the horizontal eye level are more pronounced when the ocular muscles are curarized, but only in the absence of a structured visual environment (Matin et al., 1982; Matin, Stevens, \& Picoult, 1983). Moreover, for a person whose head has been tilted backward for about $2 \mathrm{~min}$ and whose eyes are therefore under the influence of the doll reflex, the subjective perception of the TPE shifts upward-that is, toward the upper part of the body. This rise in the perceived position of the TPE when the head is tilted has been called the elevation illusion (Ebenholtz \& Shebilske, 1975; Shebilske \& Karmiohl, 1978). Fogelgren and Shebilske (1979) suggest that this illusion could be explained by hypotheses about peripheral muscle physiology and the effect of the otolithic system on the eye muscles. Recent research contributes additional arguments supporting the existence of a relationship between the position of the eye in the socket relative to the head and the VPEL (Cohen, Ebenholtz, \& Linder, 1995).

Gravito-inertial effects on the VPEL have also been evidenced in the oculogravic illusion (Clark \& Graybiel, 1951; Cohen, 1973; Correia et al., 1968; Schöne, 1964). When an upright subject faces toward the center of a centrifuge that rotates at a steady velocity for some time, the increase in magnitude and the displacement of the gravitoinertial force $(\mathrm{Gi})$ lowers the VPEL (Figure 1C). This phenomenon has been explained by an illusory perception of body tilt in pitch and/or by a mechanical action on the otolithic organs (Miller \& Graybiel, 1966; Schöne, 1964; Wade \& Schöne, 1971). Raphel and Barraud (1994) have shown that lowering of the VPEL is also observed for very low variations of Gis $\left(9.81001 \mathrm{~m} / \mathrm{sec}^{2}\right)$ produced by radial acceleration - that is, $0.01 \mathrm{~m} / \mathrm{sec}^{2}$ and above. Moreover, there is a logarithmic relationship between very low radial acceleration and lowering of the VPEL, whereas for higher $G i$ magnitudes and tilts (i.e., $G i \geq 1.25 G$ [the gravitation force]), the relationship between radial acceleration and VPEL is linear (Cohen, 1973; Correia et al., 1968).

Raphel and Barraud (1994) suggest that the differences between the linear function (higher $G i$ magnitudes and tilts) and the logarithmic function (lower radial accelerations) may be caused by differences in sense organ stimulation. Indeed, when Gis are strong, the effects of oculogravic illusion are due not only to the influence of the changes in the shearing forces on the utricular and the saccular otolithic membranes, but also to the influence of tactile sensations, kinesthetic sensations, and, sometimes, muscular efforts required to maintain the body in its posture. On the other hand, when radial accelerations are very low, bodily senses - affected by the lowest Gisare restricted, and, under such conditions, lowering of the VPEL is probably due to stimulation of the otolithic system alone. Raphel and Barraud hypothesize that the utricular membrane may be especially responsible for lowering the VPEL.

In an attempt to develop a specific hypothesis on the contribution of the otolithic system to the elaboration of visual perception of subjective references in complete darkness, we have undertaken a study investigating the effects of low radial accelerations produced by centrifugation on the VPAZ - that is, subjects in a supine position-in which, by comparison with the upright position, the vestibular system is rotated $90^{\circ}$ with respect to $G$ (Figure 2). Thus, in the supine position, the shearing forces of centrifugation act especially on the saccular membrane, whereas in the upright position, they act on the utricular membrane. Under these conditions, do low radial centrifugation-induced accelerations produce the same effects on the VPAZ (see Figure 2) as those observed in

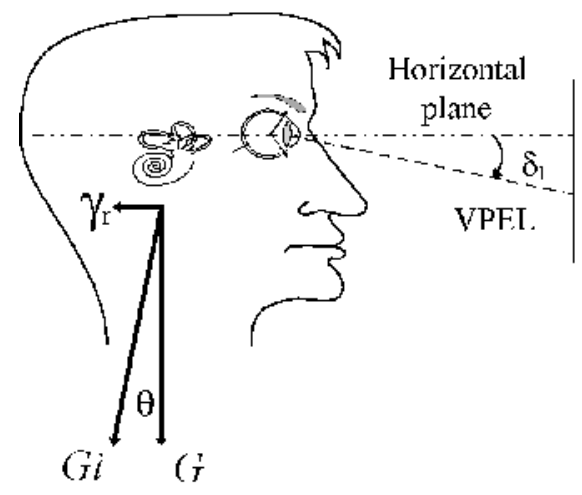

(Kaphel \& Barattd, [994!

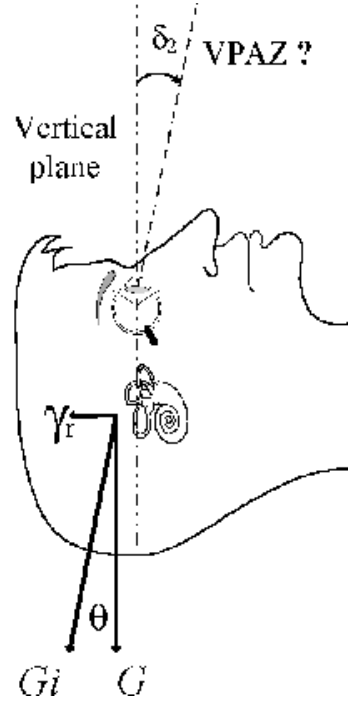

Figure 2. Positions of the vestibular system relative to the conditions of centrifugation: in the upright position for the left-hand side of the diagram and in the supine position for the right-hand side. 
the upright position for VPEL-that is, a shift of the VPAZ in the same direction as that in which the resultant Gis are shifted?

\section{METHOD}

\section{Subjects}

Twelve male subjects participated in this study. Their ages ranged from 23 to 37 years (median, 30.5; mean, 32.4; $S D$, 9.4), their body mass ranged from 64 to $79 \mathrm{~kg}$ (mean, 70.8; $S D, 4.3$ ), and their heights ranged from 165 to $183 \mathrm{~cm}$ (mean, 173.9; $S D, 6.6$ ). Their eyesight was normal or corrected to normal, and they were in good healththat is, no vestibular disorders or motion sickness symptoms. Only male subjects were included, for the sake of comparison with the study conducted 3 years ago by Raphel and Barraud (1994), in which only male subjects took part. The experiment was carried out with their signed informed consent in compliance with the Huriet Law (i.e., Helsinki Convention), which governs and regulates human experimentation in France.

\section{Apparatus}

The basic apparatus (Figure 3) was a rotating horizontal plane (centrifuge) that could rotate at a constant velocity between $0 \% \mathrm{sec}$ and $180^{\circ} / \mathrm{sec}$ around the chest to spine $(\mathrm{Gx})$ axis of the supine subjects. The subjects faced the zenith with the external auditory meatus $50 \mathrm{~cm}$ from the axis of rotation. When the horizontal plane rotated, the resultant $G i$ applied to the head of the subject was the vectorial sum of the radial acceleration $\gamma_{\mathrm{r}}$ combined with $G$, with $G i$ greater than $G$ and tilted with respect to the vertical (Figures 2 and 3).

There was a luminous target $30 \mathrm{~cm}$ in front of the subject, which was a yellow light-emitting diode (LED) fixed on the pencil holder of an electronic plotting board in a horizontal position, with a displacement accuracy of $0.05 \mathrm{~mm}$. The brightness of the LED $\left(2.82 \mathrm{~cd} / \mathrm{m}^{2}\right.$ measured by Spectrascan 650$)$ was adjusted to be perceptible without dark accommodation. Moreover, installed in a darkroom laboratory, all the elements of the basic apparatus and the electronic plotting board were constructed and painted black so that,

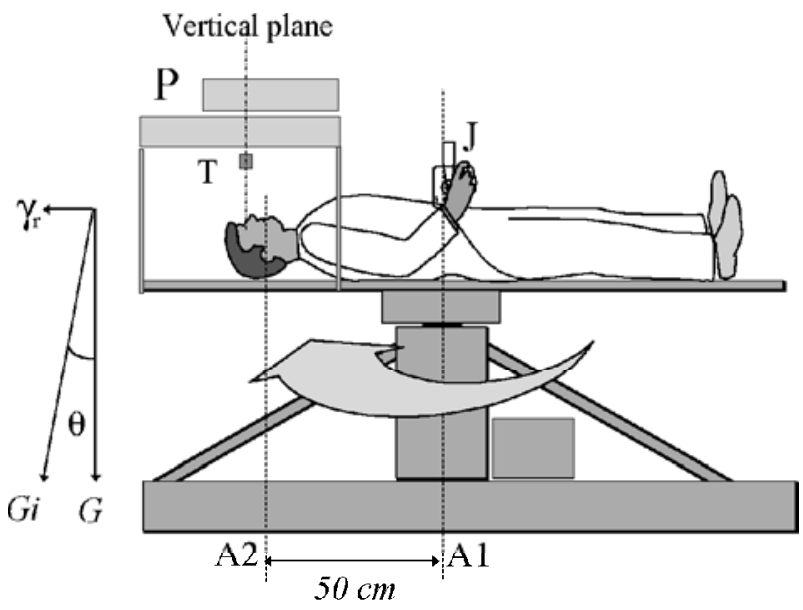

Figure 3. The apparatus consists of a yaw rotating horizontal plane (centrifuge). The illustration shows the distance between the axis of rotation (A1) and the axis of the subject's ear (A2), the electronic plotter board (P), the luminous target as a light-emitting diode $(T)$, the joystick $(J)$, and the vertical plane through the eyes and the initial position of the light-emitting diode. Also shown is the vectorial sum $G i$ between $G$ and radial acceleration $\gamma_{\mathrm{r}}$ under centrifugation. $G i$ is tilted at an angle with respect to the vertical. when the LED was switched on, the subjects did not perceive any visual reference. With respect to the body references, the subjects could move the LED both "above" versus "below" and "to the right" versus "to the left," using a joystick. LED motions on the electronic plotting board and centrifuge rotation velocity were computer controlled and monitored.

\section{Experimental Design}

The experiment was run under six experimental conditions. For the six conditions, the angular velocities were $0 \% \mathrm{sec}$ (motionless), $60 \% \mathrm{sec}, 80 \% \mathrm{sec}, 100 \% \mathrm{sec}, 120 \% \mathrm{sec}$, and $140 \% \mathrm{sec}$, corresponding to radial accelerations of $0,0.55,0.97,1.52,2.19$, and $2.98 \mathrm{~m} / \mathrm{sec}^{2}$. The data of radial accelerations, Gis, and tilted angles of $G i$ with respect to $G$ are shown in Table 1 . Each of the conditions was run in a separate session. Separate VPAZ measurements were made during each gravito-inertial condition and following each gravitoinertial condition. In addition, at the beginning of each session, prior to beginning rotation, an additional motionless set of measurements was made; these will be referred to as the reference VPAZ. For each subject, the six experimental sessions were carried out in a randomized order and separated by a minimum of 2 days.

\section{Procedure}

Just before each session, the subjects were instructed to use the joystick to set, in total darkness, the luminous target at the place perceived as the VPAZ. For every setting, the target was switched off and randomly displaced-both the $x$ - and the $y$-axes-in the periphery of the electronic plotting board. The LED was then switched on, and a pip sound gave the signal to set the target to the VPAZ and to validate the setting with a trigger on the joystick. The subjects made four trials for each VPAZ measurement. There were two reasons for displacing the LED on the $x$ - and $y$-axes: (1) to randomly position the LED in a large visual field at the beginning of each setting of the VPAZ - that is, not staying only on the $y$-axis; and (2) to allow the subject to make a precise setting of the VPAZ, which was not necessarily in the physical sagittal plane between the eyes.

When the subject was lying down on the rotating horizontal plane, the middle of the plotter (zero on the $x y$ axis) was brought into alignment with the vertical median plane through the bridge of the nose (between the eyes). Comfortably settled and held motionless on the rotating horizontal plane, the subject was further instructed to keep his head steady against the headrest at all times. Fifteen seconds after the LED was set for the subject, the laboratory lights were switched off, and the subject started each gravito-inertial condition by making four trial settings of the VPAZ as reference values, while remaining motionless and in total darkness (Figure 4). The centrifuge was then started up slowly (except for the motionless gravito-inertial condition), and after 2 min of either rotation at constant velocity or being motionless, the subject was asked to make four settings to the VPAZ under this gravito-inertial condition. Finally, the centrifuge was stopped gradually (except for the motionless gravito-inerti al condition), and after being motionless for 2 min, the subject again made four control settings to the VPAZ. Each trial for setting the VPAZ lasted about $20 \mathrm{sec}$, and each experimental session lasted about 15 min.

\section{Data Collection}

All the measurements were made with respect to the center of the plotter (zero on the $x y$ axis). For each situation (motionless or with rotation), the VPAZ was averaged over the four trials. However, since the experimental effects expected on the VPAZ concerned the $y$-axis, only the measurements made on this axis were taken into account in the data processing. For each of the six experimental conditions, the starting VPAZ, measured while the subject was motionless and in total darkness, was compared with zero on the $x y$ axis of the plotter and served as a reference value, $\delta_{1}$. Experimental VPAZ data in centrifugation conditions consisted of the algebraic differ- 
Table 1

Experimental Gravito-Inertial Conditions Under Centrifugation

\begin{tabular}{cccc}
\hline Rotation Velocity & $\begin{array}{c}\text { Radial Accelerations } \gamma_{\mathrm{r}} \\
\left(\mathrm{m} / \mathrm{sec}^{2}\right)\end{array}$ & $\begin{array}{c}\text { Gravito-Inertial Force } \\
\text { Gi }\left(\mathrm{m} / \mathrm{sec}^{2}\right)\end{array}$ & $\begin{array}{c}\text { Tilted Angle } \theta \text { of } G i \\
\text { With Respect to G (degrees) }\end{array}$ \\
\hline Motionless & 0 & 9.810 & 0 \\
$60 \% \mathrm{sec}$ & 0.55 & 9.825 & 3.21 \\
$80 \% \mathrm{sec}$ & 0.97 & 9.858 & 5.64 \\
$100 \% \mathrm{sec}$ & 1.52 & 9.927 & 8.81 \\
$120 \% \mathrm{sec}$ & 2.19 & 10.050 & 12.60 \\
$140 \% \mathrm{sec}$ & 2.98 & 10.250 & 16.90 \\
\hline
\end{tabular}

ence $\varepsilon_{2}$ between the VPAZ measured under gravito-inertial conditions $\left(\delta_{2}\right)$ and the VPAZ reference value $\left(\delta_{1}\right): \varepsilon_{2}=\delta_{2}-\delta_{1}$. VPAZ control with the centrifuge at rest (after centrifugation) consisted in the algebraic difference $\varepsilon_{3}$ between the motionless VPAZ measurements after $\left(\delta_{3}\right)$ and before $\left(\delta_{1}\right)$ centrifugation: $\varepsilon_{3}=\delta_{3}-\delta_{1}$. When $\varepsilon$ was negative, the VPAZ was below the VPAZ reference value (toward the lower part of the body) and when it was positive, the VPAZ was above the VPAZ reference value (toward the upper part of the body). The VPAZ data were expressed in degrees of visual angle. Data selected for statistical processing were $\delta_{1}$ for the VPAZ reference, $\varepsilon_{2}$ for VPAZ under the six gravito-inertial conditions, and $\varepsilon_{3}$ for VPAZ control after centrifugation. The head of the subject being motionless during all the experimental sessions, the relative measurement $\varepsilon$ was selected to remove any inaccuracy that might have been introduced as the subject was being installed on the apparatus-namely in terms of aligning with great precision the center of the plotter onto the gaze axis (nose bridge).

\section{RESULTS}

\section{Vertically Perceived Apparent Zenith With \\ Subjects Motionless $\left(\delta_{1}\right)$}

The mean of the VPAZ reference values $\left(\delta_{1}\right)$, measured with subjects in the supine position and motionless in total darkness at the beginning of each experimental session, was globally above the vertical plane through the eyesthat is, toward the upper part of the head (mean $\delta_{1}=0.62^{\circ}$ of visual angle, $S D=3.59^{\circ}$ ). A one-way analysis of variance (ANOVA) showed no significant difference in the VPAZ references between the six sessions $[F(5,55)=$ $0.72, p>.6$; Table 2] and no between-subjects effect $[F(1,11)=0.76, p>.4]$.

Moreover, for the experimental condition with the centrifuge at rest, there were no significant differences between the VPAZ reference $\left(\delta_{1}\right)$ and (1) the VPAZ of the motionless gravito-inertial condition $\left(\varepsilon_{2}\right)$, assessed 2 min after the settings of the VPAZ reference (paired $t$ test $=0.06$, n.s.), and (2) the VPAZ control after centrifugation $\left(\varepsilon_{3}\right)$, assessed less than 10 min after the VPAZ reference (paired $t$ test $=0.98$, n.s.).

\section{Vertically Perceived Apparent Zenith With Subjects Under Low Centrifugation $\left(\boldsymbol{\epsilon}_{2}\right)$}

The VPAZ perceived under centrifugation was lower than the reference VPAZ - that is, shifted upward toward the lower part of the body (Table 2; Figure 5). Moreover, a one-way ANOVA showed a significant difference between the gravito-inertial conditions (i.e., 0, 0.55, 0.97, $1.52,2.19$, and $2.98 \mathrm{~m} / \mathrm{sec}^{2}$ ) on the variation settings of the $\operatorname{VPAZ}[F(5,55)=3.305, p=.011]$, as well as a significant between-subjects effect $[F(1,11)=9.729, p=.01]$.

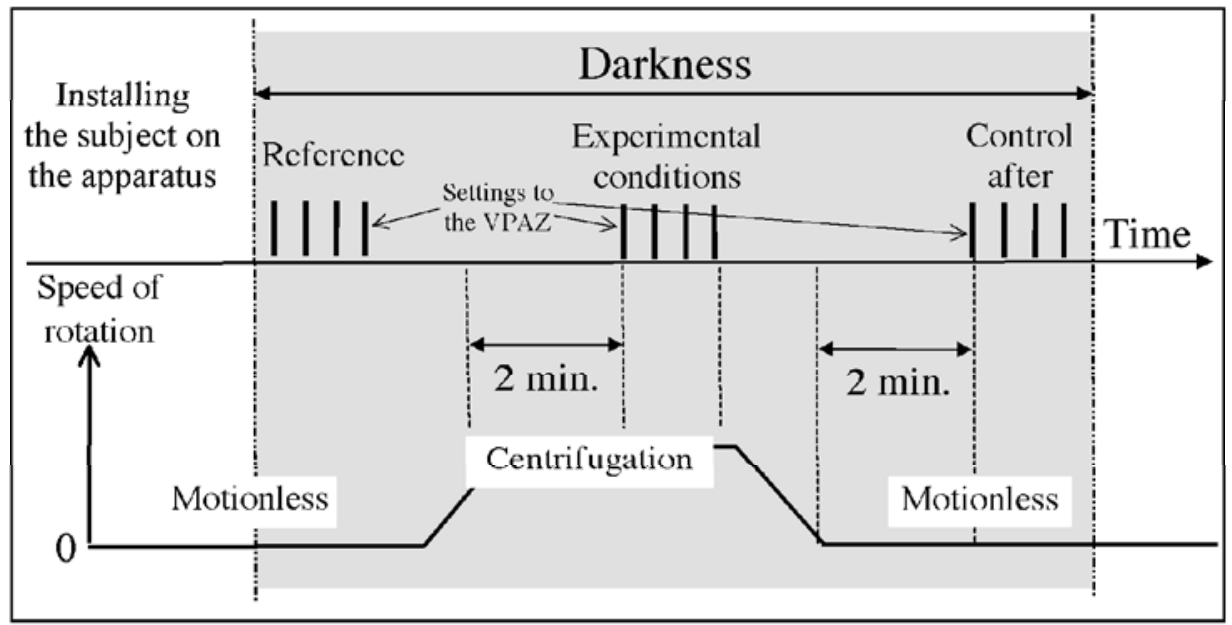

Figure 4. The graph shows the experimental design. After the subject is installed on the apparatus, the session consists of three phases in darkness. The first is for measuring the visually perceived apparent zenith (VPAZ) reference, the second is the centrifugation condition, and the last is the control phase while motionless. For each phase, there are four settings to the VPAZ. After the centrifuge is slowly started up or gradually stopped, there is a 2 -min wait for stabilization before beginning the settings to the VPAZ. 
Table 2

Average Deviations of Visually Perceived Apparent Zenith (VPAZ) Settings for the Motionless Condition Relative to the Gravitational Vertical (i.e., VPAZ reference $\left.\delta_{1}\right)$, for the Centrifugation Conditions $\left(\epsilon_{2}\right)$ Relative to the VPAZ Reference, and for the Motionless Control Conditions After Centrifugation $\left(\epsilon_{\mathbf{3}}\right)$ Relative to the VPAZ Reference

\begin{tabular}{|c|c|c|c|c|c|c|c|c|c|c|c|c|}
\hline \multirow[b]{2}{*}{ VPAZ } & \multicolumn{2}{|c|}{ Motionless } & \multicolumn{2}{|c|}{$0.55 \mathrm{~m} / \mathrm{sec}^{2}$} & \multicolumn{2}{|c|}{$0.97 \mathrm{~m} / \mathrm{sec}^{2}$} & \multicolumn{2}{|c|}{$1.52 \mathrm{~m} / \mathrm{sec}^{2}$} & \multicolumn{2}{|c|}{$2.19 \mathrm{~m} / \mathrm{sec}^{2}$} & \multicolumn{2}{|c|}{$2.98 \mathrm{~m} / \mathrm{sec}^{2}$} \\
\hline & VPAZ & $S D$ & VPAZ & $S D$ & VPAZ & $S D$ & VPAZ & $S D$ & VPAZ & $S D$ & VPAZ & $S D$ \\
\hline $\begin{array}{l}\text { Reference while motionless, } \delta_{1} \\
\text { Deviations under centrifugation, } \varepsilon_{2} \\
\text { Deviations in motionless control, } \varepsilon_{2}\end{array}$ & $\begin{array}{r}-0.35 \\
-0.09 \\
1.56\end{array}$ & $\begin{array}{l}3.03 \\
5.06 \\
5.55\end{array}$ & $\begin{array}{r}1.12 \\
-0.66 \\
1.27\end{array}$ & $\begin{array}{l}3.08 \\
3.02 \\
3.05\end{array}$ & $\begin{array}{r}0.23 \\
-2.57 \\
2.06\end{array}$ & $\begin{array}{l}5.02 \\
3.67 \\
4.59\end{array}$ & $\begin{array}{r}0.68 \\
-3.91 \\
3.21\end{array}$ & $\begin{array}{l}2.98 \\
4.88 \\
5.52\end{array}$ & $\begin{array}{r}1.71 \\
-4.34 \\
4.59\end{array}$ & $\begin{array}{l}4.13 \\
4.11 \\
4.76\end{array}$ & $\begin{array}{r}0.36 \\
-4.80 \\
4.39\end{array}$ & $\begin{array}{l}1.96 \\
6.09 \\
6.44\end{array}$ \\
\hline
\end{tabular}

Note-Values and standard deviations $(S D)$ are given in degrees of visual angle.

In addition, in the five centrifugation conditions (i.e., $0.55,0.97,1.52,2.19$, and $\left.2.98 \mathrm{~m} / \mathrm{sec}^{2}\right)$, a significant relationship was found between radial acceleration $\gamma_{\mathrm{r}}(\mathrm{Ta}-$ ble 1) and the lowering of the VPAZ (Table 2). This relationship is described by the logarithmic regression equation: $D \gamma_{\mathrm{r}}=-2.467 \mathrm{Ln} \gamma_{\mathrm{r}}-2.424\left(R^{2}=.96\right.$, $p=.003$ ), where $D \gamma_{\mathrm{r}}$ (in degrees) is the decrease in the visual angle of the VPAZ, relative to the VPAZ reference measured while motionless, and $\gamma_{\mathrm{r}}$ (in meters per seconds squared) is the radial acceleration of centrifugation (Figure 6).

\section{Visually Perceived Apparent Zenith With}

\section{Subjects Motionless After Centrifugation $\left(\epsilon_{3}\right)$}

Whereas the VPAZ was lowered under centrifugation, the VPAZ measured after centrifugation rose, with respect to the VPAZ reference, toward the upper part of the body (Table 2; Figure 5). However, there was no significant difference on the settings of the VPAZ after-control condition between the six gravito-inertial conditions (i.e., $0,0.55$, $0.97,1.52,2.19$, and $\left.2.98 \mathrm{~m} / \mathrm{sec}^{2}\right)[F(5,55)=1.359, p=$ .25], whereas there was a significant between-subjects effect $[F(1,11)=8.967, p=.012]$.

Moreover, for the $0,0.55,0.97$, and $1.52 \mathrm{~m} / \mathrm{sec}^{2}$ conditions, there was no significant difference between the VPAZ reference $\delta_{1}$ and the VPAZ control after centrifugation $\varepsilon_{3}$ [paired $t$ test $\leq 2.02, p>.065$ ]. However, for the 2.19 and $2.98 \mathrm{~m} / \mathrm{sec}^{2}$ conditions, there was a significant difference between $\delta_{1}$ and $\varepsilon_{3}$ (paired $t$ test: 3.33 , $p=.007$, and 2.35, $p=.039$, respectively).

Finally, for each of the five conditions with centrifugation, mean deviations of VPAZ ( $\varepsilon_{2}$ and $\varepsilon_{3}$ ) differed significantly between centrifugation and motionless control after centrifugation (paired $t$ test $\geq 2.40, p \leq .035$; see Figure 5 for $p$ values). Moreover, the more the VPAZ was lowered by centrifugation, the more the VPAZ control after centrifugation was raised (Spearman correlation $r=-.90$, $p=.037$; Table 2 and Figure 5).

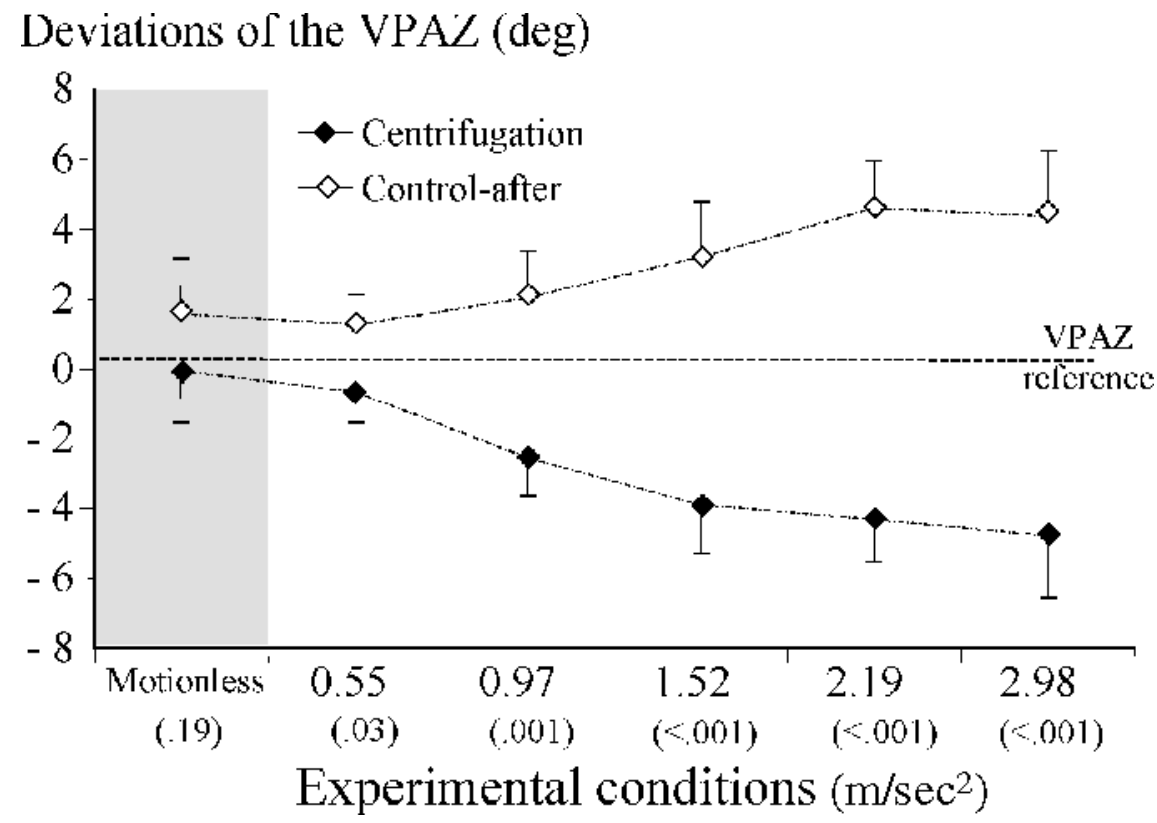

Figure 5. The graph plot deviation of the visually perceived apparent zenith (VPAZ) settings according to VPAZ reference in the gravito-inertial conditions (motionless and five acceleration modes) and for control after centrifugation in motionlessness. Zero on the $y$-axis is the VPAZ reference (motionless in total darkness). For each gravito-inertial mode, including motionlessness, the graph shows the $p$ values of the paired $t$ test obtained for each centrifugation condition and its matching VPAZ motionless after-control condition. 


\section{Deviations of the subjective reference $D \gamma_{\mathrm{r}}(\mathrm{deg})$}

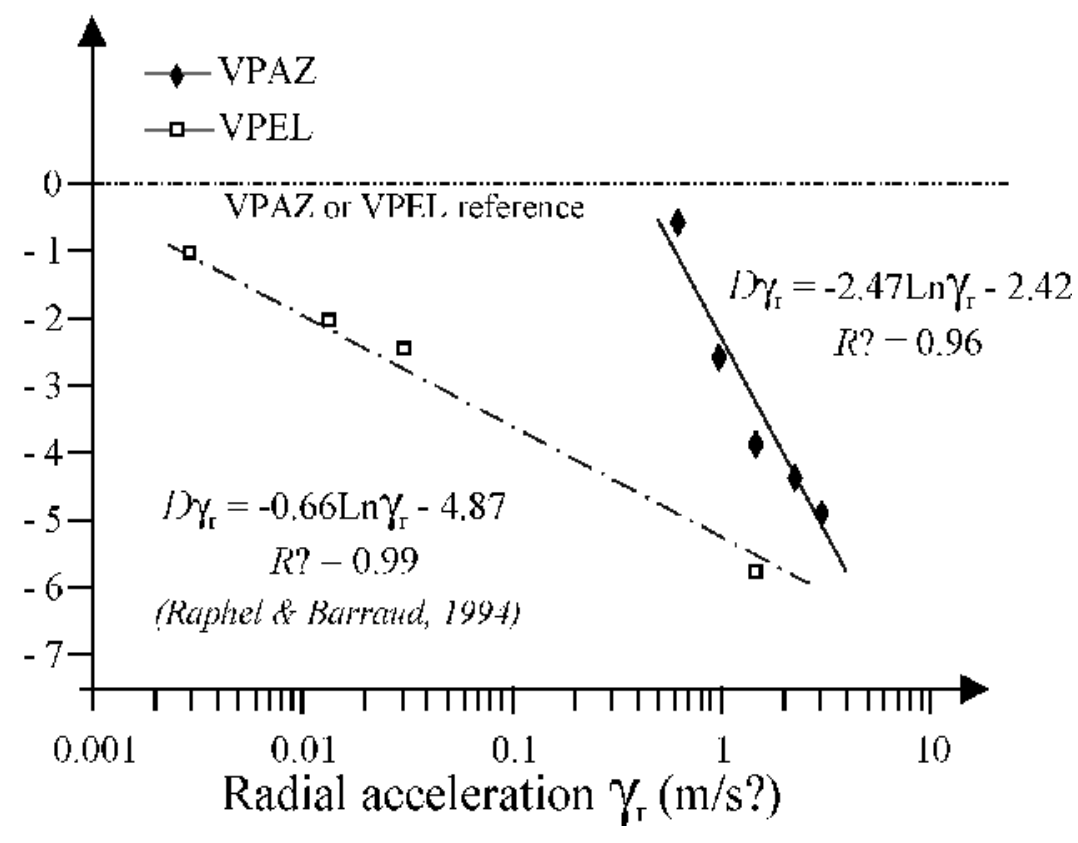

Figure 6. The graph plots the lowering of the visually perceived apparent zenith (VPAZ, $\left.D \gamma_{r}\right)$ as a logarithmic function of radial acceleration magnitude $\left(\gamma_{\mathrm{r}}\right)$ under low centrifugation. By comparison, there is the logarithmic function regression of the visually perceived eye level (VPEL) in a seated position (i.e., body upright) as observed by Raphel and Barraud (1994). Zero on the $y$-axis is the visually perceived reference value for both curves (VPAZ and VPEL), while motionless and in darkness.

\section{DISCUSSION}

The first interesting finding is the difference between the results observed in this study concerning the settings of VPAZ at the beginning of each experimental session in total darkness and those described by Cohen and Larson (1974) concerning the perception of the morphological horizon. It should be noted that the VPAZ is referenced to the external space, whereas the morphological horizon is referenced to an imaginary plane, through the center of the eyes, that runs perpendicular to the longitudinal body axis. Thus, for these authors, the morphological horizon was "below" the gravitational vertical (i.e., in the direction of the lower part of the body), whereas in this study, the VPAZ was "above" the gravitational vertical (i.e., in the direction of the upper part of the body). The results also differ in intensity, since the gap between the true gravitational vertical and the morphological horizon was in the $10^{\circ}$ range for Cohen and Larson, whereas VPAZ error was below $1^{\circ}$ in this study. Even if the two experiments measured different but closely related percepts, the differences observed may be explained by the manner in which the subjects were put in the supine position. Cohen and Larson pitched the subjects in complete darkness without visual reference, starting from the upright position. This pitch rotation causes an doll eye reflex (Citek \& Ebenholtz, 1996), which can have a determining influence on the subjective perception of the morphological horizon, as was pointed out by Shebilske and Fogelgren (1977) and Fogelgren and Shebilske (1979). In our study, the subjects were installed on the horizontal plane, as if lying on a bed, in an illuminated environment. Under these conditions, the subjects had additional visual references to calibrate their spatial perception of the environment from the supine position. These experimental differences might account for the differences in the results obtained in the two studies. Again, this emphasizes the disparity of previous research concerning the orientation of the head, relative to gravity, in the perception of spatial references, which is strongly conditioned by the experimental design under which the extraretinal perceptive systems are investigated.

The experimental design closest to that in this study was reported by Raphel and Barraud (1994) and Raphel et al. (1996), since in all cases, subjects were motionless in complete darkness and carried out the settings of the VPEL or the VPAZ a few seconds after extinction of the light. The comparison of results shows a notable difference between the upright and the supine positions of the body in setting the visually perceived subjective references. Indeed, they differ in the direction of the deviation, since the VPEL in total darkness is "below" the transverse eye level by $3^{\circ}-4^{\circ}$ (Raphel \& Barraud, 1994; Raphel et al., 1996), whereas in the supine position, the VPAZ is "above" the 
true gravitational position (about $0.6^{\circ}$ ). In these studies, which differ only by the head position in the gravitational field, the perceptive differences concern (1) the vestibular system, which is rotated $90^{\circ}$, and (2) the neck muscles, which actively maintain the head in the upright position and are slackened in the supine position. Although the respective contribution of this sensory information in the subjective processing of the VPEL and the VPAZ cannot be defined, the observed upright versus supine differences confirm the role of extraretinal information regarding the orientation of the head relative to gravity, as was suggested by Stoper and Cohen (1986).

Finally, concerning the VPAZ reference measured with subjects in the supine position and motionless in total darkness, it is important to emphasize the homogeneity of the measurements, especially for the VPAZ reference value between the six experimental sessions. Thus, the results obtained from motionless subjects in total darkness demonstrated the stability and the validity of the experimental design, as well as the lack of betweensubjects variability.

The second interesting finding is the significant lowering, relative to the body axis, of the VPAZ induced by low Gis (centrifugation) with subjects in a supine position and total darkness. Before commenting on these results, it is important to emphasize that under very low centrifugation, the subjects did not experience any sensation of disorientation or dizziness. Especially, at a steady velocity in darkness, they perceived neither the motion of rotation nor the corresponding gravito-inertial variations of $G i$; that is, the subjects were completely unaware that the VPAZ became lower in relation to Gis. Thus, by comparison with initial results observed in the upright po- sition (Raphel \& Barraud, 1994), this experiment shows that low radial accelerations also result in a VPAZ shift in the same direction as the shift of the resultant $G i$ (Figure 2). However, there is an amplification or an attenuation of the deviation of the eye level according to both the body position (upright vs. supine) and the angular deviation of $G i$. Indeed, Raphel and Barraud show that for $G i$ shifted by $\theta \leq 1.95^{\circ}$, the corresponding VPEL shift is greater than the $G i$ shift (i.e., amplification), whereas for a $9.6^{\circ} \mathrm{Gi}$ shift, the corresponding VPEL shift is lower (i.e., attenuation; Figure 7). In this study, the VPAZ shift was always lower than the $G i$ shift (i.e., attenuation), in agreement with data that suggest a head-tilt-dependent drop in otolithic sensitivity (Howard, 1982, 1986; Schöne, 1964, 1975; Udo de Haes, 1970; Wade \& Schöne, 1971). However, the data illustrated in Figure 7 would indicate that about $2^{\circ}-3^{\circ}$ of angular deviation of $G i$, relative to $G$, the VPAZ shift, like the VPEL shift, corresponds roughly to the theoretical data. Further work would be necessary to check this point.

Furthermore, it is interesting to note that in the range of very weak gravito-inertial stimulation used in this study and by Raphel and Barraud (1994), perception of the eye level corresponds to a logarithmic psychophysical function, but with different values for the constants between the supine and the upright positions (Figure 6). Indeed, the theoretical threshold of sensitivity to radial accelerations is higher in the supine position $\left(0.38 \mathrm{~m} / \mathrm{sec}^{2}\right)$ than in the upright position $\left(0.0006 \mathrm{~m} / \mathrm{sec}^{2}\right)$. Conversely, VPAZ variations as a function of radial accelerations were higher (therefore, more noticeable) than VPEL variations - that is, the lowering of the eye level varied more when the subjects were lying down than when they were in an upright

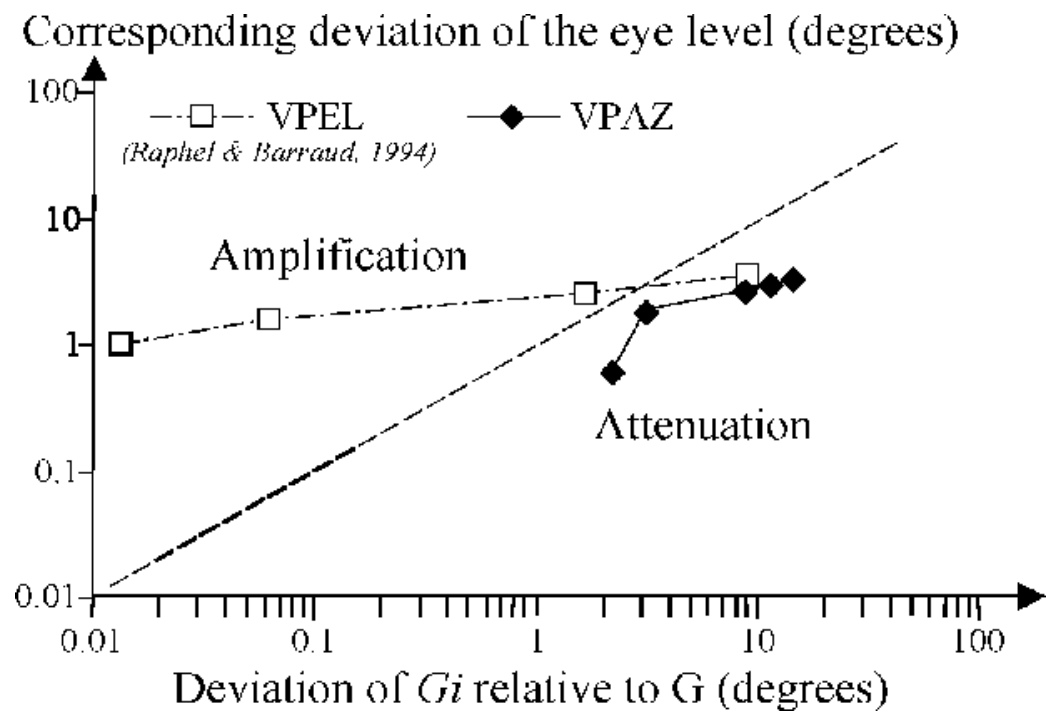

Figure 7. The graph plots the relation between the deviation $\theta$ of the gravito-inertial force $G i$, relative to $G$, and the corresponding deviation of the eye level relative to the eye level reference value. The visually perceived apparent zenith (VPAZ) is always attenuated whatever $\theta$ is, whereas the visually perceived eye level (VPEL; Raphel \& Barraud, 1994) is amplified with $\theta \leq \mathbf{1 . 9 5}^{\circ}$ and attenuated for the $\theta$ value above. In order to permit the representation of the two functions (VPAZ and VPEL), the scales are logarithmic. 
position (Figure 6). It is noteworthy to observe not only the highly significant relation between the radial acceleration and the lowering of the eye level, but especially the great differences between the two logarithmic psychophysical functions, although we took extreme precautions in order to reproduce the same experimental design in the supine position as in the upright position.

Another interesting finding concerns the significant difference between the subjects observed in all conditions of centrifugation and motionless controls after centrifugation. A Spearman correlation between the VPAZ values of the three conditions of measurement $\left(\delta_{1}, \varepsilon_{2}\right.$, and $\left.\varepsilon_{3}\right)$, shows a link only between the VPAZ under centrifugation $\varepsilon_{2}$ and the VPAZ control $\varepsilon_{3}$ in motionlesness after centrifugation $(r=.48, p<.001)$. These data suggest a variability between the subjects in sensitivity to very low radial accelerations, a variability whose effects are reflected after centrifugation in the motionless condition.

In sum, these results and previous research support the notion that (1) eye level perception in darkness takes into account the changes, even if extremely slight, in the direction of the $G i$ produced by the combination of gravity and radial acceleration, (2) for low radial accelerations produced by centrifugation, threshold sensitivity and responses in the subjective eye level perception vary according to body position relative to gravity, and (3) responses in the subjective eye level perception in a supine position vary according to the subject's sensitivity to low radial accelerations produced by centrifugation.

To understand the mechanisms of these psychophysical functions, let us use the speculative explanations proposed by Raphel and Barraud (1994), whereby the responsibility for the phenomenon of lowering of the VPEL is assigned to the utricular membrane for three reasons. First, the very limited variations of the resultant $G i$ exclude any tactile and kinesthetic origin, since the variations in subject's weights are but a few hundred milligramsthat is, negligible relative to the threshold sensitivities of the tactile and kinesthetic organs. Second, even if the anatomical structure and spatial position of the otolithic system is complicated, in accordance with Kelly (1991) and Citek and Ebenholtz (1996), we can affirm that the utricular membrane has an orientation close to the horizontal plane when the head is held erect, whereas the saccular maculae is globally oriented nearly vertically. Third, with very low radial acceleration in the upright position, the shearing force variations in the vertical direction are negligible (ranging from $0.001 \%$ to $1.3 \%$ ), whereas shearing force variations produced by the variation of radial acceleration in the horizontal direction are very important (ranging from $140 \%$ to $4,900 \%$ ).

In this study, weight variations as a function of centrifugation in the supine position make for a much more complex situation, because of the gradient of Gis applied to the body. The gradient decreases from the head to the part of the thorax located on the rotation axis of the centrifuge, where $G i=G$ (i.e., $9.81 \mathrm{~m} / \mathrm{sec}^{2}$ ); it then increases to reach its maximum on the feet. Under such conditions, it is difficult to determine the effective weight variations based on the morphology of the subjects and the Gis. Let us say that, for a subject weighing $70 \mathrm{~kg}$ and measuring $1.70 \mathrm{~m}$, the theoretical increase in weight under the fastest centrifugation condition (i.e., $G i=10.25 \mathrm{~m} / \mathrm{sec}^{2}$ ) is evaluated to be less than $1 \mathrm{~kg}$, distributed over the posterior aspect of the body from head to feet. This is negligible for tactile perception. Thus, in parallel to the notion that the utricular membrane is assumed to be involved in the upright position (Raphel \& Barraud, 1994), the saccular membrane would be involved in the supine position, since it undergoes higher shearing force variations than does the utricular membrane. Indeed, whereas shearing force variations applied in a plane close to that of the utricular membrane range from $0.15 \%$ to $4.5 \%$, shearing force variations applied in a plane close to that of the saccular membrane range from $36 \%$ to $441 \%$.

However, how do we account for the functional anisotropy in the setting of the eye level in darkness, under radial accelerations, between an upright and a supine position? Two speculative functional assumptions can be proposed, one peripheral and the other one central. According to the literature that hypothesizes that the sensitivity of the otolithic organs and its effects on spatial perception vary with the tilt of the whole body (Howard, 1982, 1986; Schöne, 1964, 1975; Udo de Haes, 1970; Wade \& Schöne, 1971), the first explanation of the peripheral assumption supposes a significant functional difference between the neurophysiological properties of the saccular membrane and those of the utricular membrane. It should be noted that the only relevant neuroanatomical and histophy siological argument currently suggested by the literature (Correia et al., 1968) is the difference in the number of sensory cells between the utricular membrane $(33,000)$ and the saccular membrane $(19,000)$. These data are in agreement with the difference in the threshold sensitivity to radial accelerations between the upright and the supine positions. The second explanation of the peripheral assumption relates to interrogations concerning the role of ocular proprioception in setting the VPAZ, according to the fact that proprioceptive and kinesthetic data from the eyes, which depend on the variations in muscle loading, play an essential part in the visual space perception of objects (Cohen et al., 1995). In the supine position, two functional levels should be considered, since the psychophysical function of the VPAZ can be regarded as the resultant of the combined effects of Gis and the vestibular system on the eye position. First, we could hypothesize that inertial forces can affect the ocular muscles by a direct mechanical action on the eyeball. However, there is a contradiction between the raising of the VPAZ observed in motionlessness and the lowering of the VPAZ observed under centrifugation-that is, in the opposite direction of the hypothetical inertial attraction of the eyeball. Secondly, very low gravito-inertial stimulation should bring into play a mechanical reflex action of the vestibular system on the position of the eye in the socket. This effect could be a doll reflex with a rotation of the eye 
toward the bottom part of the body - that is, correlatively with the displacement of $\mathrm{Gi}$-which could explain the lowering of the VPAZ under centrifugation. This assumption would also account for the significant aftereffect observed with the centrifuge at rest after centrifugation under 2.19 and $2.98 \mathrm{~m} / \mathrm{sec}^{2}$ radial accelerations. This effect is similar to that observed after extended exposure to hyper- or hypogravity (Cohen, 1970; Welch, Cohen, \& deRoschia, 1996) or to the elevation illusion interpreted by hypotheses about eye muscle physiology (Fogelgren \& Shebilske, 1979). Whatever the relevance of the explanations suggested by these functional assumptions, a strictly peripheral and sensory explanation is not satisfactory in accounting for the psychophysical phenomena observed in upright versus supine body positions. Indeed, how can the functional contradiction between a very low perceptive threshold, associated with a low sensitivity to acceleration variations in the upright position, and a high perceptive threshold, associated with a higher sensitivity to acceleration variations in the supine position, be explained (Figure 6)?

The second speculative assumption therefore considers neuropsychological functioning in detecting and processing sensory signals. It suggests, of course, that the role of vestibular and ocular proprioceptive information on the subjective eye level perception should be analyzed and interpreted not only on the basis of the associated physical forces and sensory signals, but also in terms of the relevance assigned to the peripheral information by the integrative sensory functions and the associative processes. The general assumption is that relevance, integration, and mental processing of sensory signals all depend on the conditions under which the sensory organs are usually stimulated - that is, the functional capacities of the system are determined by the knowledge acquired through experience. The utricular membrane is primarily activated by backward and forward movements of the head under accelerations ranging from zero to several meters per seconds squared. Under such conditions, the detection threshold for vestibular signals should be very low, and the sensitivity to variations in linear backward and forward accelerations should be low, considering the magnitude of the accelerations to which the vestibular system can be subjected. The saccular membrane is continuously subjected to gravity (i.e., $9.81 \mathrm{~m} / \mathrm{sec}^{2}$ ) when subjects are standing upright, and variations in the gravitational direction usually are very small. Under such conditions, the detection threshold of linear accelerations should be higher. Likewise, the sensitivity to acceleration variations in the gravity direction should be higher, since variations are usually limited in this direction.

Obviously, these speculative explanations require complementary work in order to be validated. On a technical level, it would be interesting to use an ISCAN infrared video system, as in Cohen et al. (1995) and Citek and Ebenholtz (1996), to control gaze variations in a supine position under low centrifugation. Moreover, it would be useful to study the effects of very low centrifugationinduced Gis on the subjective perception of the TPE, using different tilted body positions between upright and supine. Furthermore, the exposure time of the vestibular system and the ocular proprioception in the gravitational field should also be studied to assess possible processing changes. The subject should be maintained in the supine position for several minutes, several hours, and even several days (i.e., bed rest), and then the VPAZ should be assessed under low centrifugation conditions. In addition, it would be useful to widen the scope of work in upright and supine body positions by using higher centrifugation values so as to gain more knowledge on the phenomena occurring between very low Gis $(1.000001 G$ to $1.045 G)$ and high Gis (1.5 to $2.0 \mathrm{G}$ ).

\section{REFERENCES}

Citek, K., \& Ebenholtz, S. M. (1996). Vertical and horizontal eye displacement during static pitch and roll posture. Vestibular Research, 6, 213-228.

Clark, B., \& Graybiel, A. (1951). Visual perception of the horizontal following exposure to radial acceleration on a centrifuge. Journal of Comparative \& Physiological Psychology, 44, 525-534.

Cohen, M. M. (1970). Sensory-motor adaptation and aftereffects of exposure to increased gravitational forces. Aerospace Medicine, 41, 318-322.

Cohen, M. M. (1973). Elevator illusion: Influences of otolith organ activity and neck proprioception. Perception \& Psychophysics, 14, 401406.

CoHEn, M. M. (1981). Visual-proprioceptive interactions. In R. D. Walk \& H. L. Pick (Eds.), Intersensory perception and sensory integration (pp. 175-216). New York: Plenum.

Cohen, M. M., Ebenholtz, S. M., \& Linder, B. J. (1995). Effects of optical pitch on oculomotor control and the perception of target elevation. Perception \& Psychophysics, 57, 433-440.

Cohen, M. M., \& Larson, C. A. (1974). Human spatial orientation in the pitch dimension. Perception \& Psychophysics, 16, 508-512.

Correia, M. J., Hixon, W. C., \& Niven, J. I. (1968). On predictive equations for subjective judgments of vertical and horizon in a force field. Acta Oto-Laryngologica, Suppl. 230, 3-20.

Ebenholtz, S. M., \& SHebilske, W. L. (1975). The doll reflex: Ocular counterrolling with head-body tilt in median plane. Vision Research, 15, 601-612.

Fogelgren, L. A., \& Shebilske, W. L. (1979). Central visual learning and illusory visual direction after backward head tilts. Perception \& Psychophysics, 25, 519-523.

HopPELER, P. (1913). Über den Stellungsfaktor der Sehrichtungen; eine experimentelle Studie [On the eye level: An experimental study]. Zeitschrift für Psychologische und Physiologische Sinnesorgane, 66, 249-262.

HowARD, I. P. (1982). Human visual orientation. New York: Wiley.

HowARD, I. P. (1986). The perception of posture, self motion, and the visual vertical. In K. R. Boff, L. Kaufman, \& J. P. Thomas (Eds.), Handbookof perception and human performance (Vol. 1, 18.1-18.62). New York: Wiley.

Howard, I. P., \& Templeton, W. B. (1966). Human spatial orientation: Orientation to gravity I (pp. 175-212). London: Wiley.

Kelly, J. P. (1991). The sense of balance. In E. R. Kandel, J. H. Schwartz, $\&$ T. M. Jessell (Eds.), Principles of neural science (3rd ed., p. 503). New York: Elsevier.

Li, W., \& MAtin, L. (1993). Eye and head position, visual pitch, and perceived eye level [Abstract]. Investigative Ophthalmology \& Visual Science, 34, 1311.

Matin, L. (1982). Visual localization and eye movements. In A. Wertheim, W. A. Wagenaar, \& H. Leibowitz (Eds.), Tutorials on motion perception (pp. 101-156). New York: Plenum.

Matin, L. (1986). Visual localization and eye movements. In K. R. Boff, L. Kaufman, \& J. P. Thomas (Eds.), Handbook of perception and human performance (Vol. 1, pp. 20.1-20.45). New York: Wiley.

Matin, L., \& LI, W. (1992). Visually perceived eye level: Changes in- 
duced by pitch-from-vertical 2-line visual field. Journal of Experimental Psychology: Human Perception \& Performance, 18, 257-289.

Matin, L., \& LI, W. (1995). Multimodal basis for egocentric spatial localization and orientation. Journal of Vestibular Research, 6, 499-518.

Matin, L., Picoult, E., Stevens, J. K., Edwards, M. W., JR., Young, D., \& MCARThUR, R. (1982). Oculoparalytic illusion: Visual field dependent mislocalizations by humans partially paralyzed with curare. Science, 216, 198-201.

Matin, L., Stevens, J. K., \& Picoult, E. (1983). Perceptual consequences of experimental extra-ocular muscle paralysis. In A. Hein \& M. Jeannerod (Eds.), Spatiallyoriented behavior (pp. 243-262). New York: Springer-Verlag.

McDougall, R. (1903). The subjective horizon. Psychological Monographs, 4 (1, Suppl. 4), 145-166.

Miller, E. F., \& Graybiel, A. (1966). Magnitude of gravito-inertial force: An independent variable in egocentric visual localization of the horizontal. Journal of Experimental Psychology, 71, 452-460.

NAIR, P. J. (1958). Relationships between postural and conative aspects of organismic state and perceptual localization of the apparent horizon. Unpublished master's thesis, Clark University, Worcester, MA.

Raphel, C., \& BarRaud, P.-A. (1994). Perceptual thresholds of radial acceleration as indicated by visually perceived eye level. Aviation, Space, \& Environmental Medicine, 65, 204-208.

Raphel, C., Barraud, P.-A., Koessler, C., \& Cian, C. (1996). Effects of a visual frame and of low radial accelerations on the visually perceived eye level. Perception \& Psychophysics, 58, 906-914.

SCHÖNE, H. (1964). On the role of gravity in human spatial orientation. Aerospace Medicine, 35, 764-772.

SCHÖNE, H. (1975). The weight of the gravity organ's signal in the control of perceptual and reflex type orientation at different body positions. Fortstritte der Zoologie, 23, 274-285.
ShaRp, W. L. (1934) An experimental study concerning visual localization in the horizontal plane. Journal of Experimental Psychology, 17, 787-797.

Shebilske, W. L., \& Fogelgren, L. A. (1977). Eye-position aftereffects of backward head tilt manifested by illusory visual direction. Perception \& Psychophysics, 21, 77-82.

SheBILSKe, W. L., \& KARMIOHL, C. M. (1978). Illusory visual direction during and after backward head tilts. Perception \& Psychophysics, 24, 543-545.

Stoper, A. E., \& Cohen, M. M. (1986). Judgments of eye level in light and in darkness. Perception \& Psychophysics, 40, 311-316.

Stoper, A. E., \& Cohen, M. M. (1989). Effect of structured visual environments on apparent eye level. Perception \& Psychophysics, 46, 469-475.

Stoper, A. E., \& Cohen, M. M. (1991). Optical, gravitational and kinesthetic determinants in judged eye level. In S. R. Ellis (Ed.), Pictorial communication in virtual and real environments (pp. 390-405). London: Taylor \& Francis.

Udo DE HAes, H.A. (1970). Stability of apparent vertical and ocular countertorsion as a function of lateral tilt. Perception \& Psychophysics, 8, 137-142.

WADE, N. J., \& SchÖNE, H. (1971). The influence of force magnitude on the perception of body position: I. Effect of head posture. British Journal of Psychology, 62, 157-163.

Welch, R. B., Cohen, M. M., \& DeRoschia, C. W. (1996). Reduction of the elevator illusion from continued hypergravity exposure and visual error-corrective feedback. Perception \& Psychophysics, 58, 22-30.

(Manuscript received September 18, 1998; revision accepted for publication April 28, 2000.) 